\title{
Boron-doped MnTe semiconductor-sensitized ZnO solar cells
}

\author{
AUTTASIT TUBTIMTAE ${ }^{\mathrm{a}, *}$, SUWANNA SHEANGLIW ${ }^{\mathrm{a}}$, KRITSADA HONGSITH $^{\mathrm{b}, \mathrm{c}}$ and \\ SUPAB CHOOPUN ${ }^{\text {b,c }}$ \\ ${ }^{a}$ Department of Physics, Faculty of Liberal Arts and Science, Kasetsart University, Kamphaeng Saen Campus, \\ Nakhon Pathom 73140, Thailand \\ ${ }^{\mathrm{b}}$ Department of Physics and Material Sciences, Faculty of Science, Chiang Mai University, Chiang Mai 50200, \\ Thailand \\ ${ }^{\mathrm{c}}$ Thailand Center of Excellence in Physics (ThEP Center), CHE, Bangkok 10400, Thailand
}

MS received 24 July 2013

\begin{abstract}
We studied the photovoltaic performance of boron-doped MnTe semiconductor-sensitized solar cells (B-doped MnTe SSCs). The B-doped MnTe semiconductor was grown on ZnO using two stages of the successive ionic layer adsorption and reaction (SILAR) technique. The two phases of B-doped semiconductor nanoparticles (NPs), i.e. MnTe and MnTe $e_{2}$ were observed with a diameter range of approximately $15-30 \mathrm{~nm}$. The result of the energy conversion efficiency of the sample with boron doping was superior compared to that of an undoped sample, due to the substantial change in the short-circuit current density and the open-circuit voltage. In addition, plots of $(\alpha h v)^{2}$ vs $h v$ with band gaps of 1.30 and $1.27 \mathrm{eV}$ were determined for the undoped and B-doped MnTe NPs, respectively. It can be noted that the boron doping effects with the change in the band gap and lead to an improvement in the crystalline quality and also intimate contact between the larger sizes of MnTe NPs. Hence, a noticeably improved photovoltaic performance resulted. However, this kind of semiconductor sensitizer can be further extended by experiments on yielding a higher power conversion efficiency and greater stability of the device.
\end{abstract}

Keywords. Manganese telluride; boron doping; successive ionic layer adsorption and reaction (SILAR); solar cell.

\section{Introduction}

The wide-band gap metal oxide, $\mathrm{ZnO}$, is one of the most important oxide semiconducting materials and has been used widely in many applications and forms many types of well-aligned one-dimensional $\mathrm{ZnO}$ nanostructures, such as photocatalysts (Georgekutty et al 2008), ultraviolet laser diodes (Okazaki et al 2011), optical switches (Mu et al 2011) and solar cells (Wang et al 2010; Majidi and Baxter 2011; Zhu et al 2011), because of its wide band gap $(\sim 3.3 \mathrm{eV})$ and exciton binding energy $(\sim 60 \mathrm{meV})$, as well its cheap price, versatility and the possibility of large scale production using non-toxic materials (Kahn et al 2005; Ravirajan et al 2006). ZnO has better electron mobility than $\mathrm{TiO}_{2}$. The mobility of $\mathrm{ZnO}$ is about 115 $155 \mathrm{~cm}^{2} \mathrm{~V}^{-1} \mathrm{~s}^{-1}$, which is much higher than that of $\mathrm{TiO}_{2}$ and which is in the order of $1 \times 10^{-5} \mathrm{~cm}^{2} \mathrm{~V}^{-1} \mathrm{~s}^{-1}$ (Ganesh et al 2009) and also has the proper energy level for effective electron transfer from semiconductor nanoparticles (NPs) to the conduction band of $\mathrm{ZnO}$ after visible region irradiation (Hu and Chen 2007; Chen et al 2010).

\footnotetext{
*Author for correspondence (tubtimtae@gmail.com)
}

However, there are major limitations because of the poor absorption in the visible to near-infrared regions. Many efforts have been made to improve the potential to absorb a wider wavelength region.

Currently, semiconductor-sensitized solar cells (SSCs) have attracted much attention because of the improved properties of nanoparticle semiconductors over their bulkier counterpart such as a high extinction coefficient (Yu et al 2003), the large intrinsic dipole moment generated from the bulk properties of the semiconductor (Vogel et al 1990, 1994), multiple exciton production per photon through the impact ionization effect over conventional dyes under high energy excitation (Beard 2011), tunable absorption owing to the quantum confinement effect by size and shape control providing an excellent tool for the design of light absorbing materials (Alvisatos 1996; Kongkanard et al 2008), high stability and resistivity against photoblenching and atmospheric oxygen/ water, and especially, the ability to be constructed as multilayered- or hybrid-sensitizers for a novel structure of semiconductor solar cells (Murray et al 2000; Kamat 2008). Most of the tailoring semiconductor sensitizers have been successfully synthesized by several groups, who have investigated characteristics and optical properties such as SnS (Miyauchi 2011), $\mathrm{Cu}_{2-x} \mathrm{~S}$ (Lin and Lee 
2011), ZnTe (Chao et al 2010), AgInS 2 (Cheng et al 2011; Hamanaka et al 2011), Ag $_{2} \mathrm{~S}$ (Tubtimtae et al 2010) and $\mathrm{Ag}_{2} \mathrm{Se}$ (Tubtimtae et al 2011).

The tailoring transition-metal tellurides represent a class of compounds which is keenly sought for opto- and thermo-electric applications as these materials have high thermoelectric power values and doping ability for $n$ - or p-types (Samal and Pradeep 2010).

Among these transition-metal tellurides, manganese telluride (MnTe) is one of the $p$-type semiconductors that we were interested in for use as a novel tailoring sensitizer because of its very high density of impurity charge carriers (Goswami and Mandale 1978), which can be used as a charge transport in a device. In addition, it has a narrow optical direct band gap of $1.3 \mathrm{eV}$ (Oleszkiewicz et al 1988), which is close to the bulk direct band gap of SnS ( $E_{\mathrm{g}} \sim 1.3-1.4 \mathrm{eV}$ ) (Potter and Simmons 1988; Panda et al 2006; Miyauchi 2011; Deepa and Nagaraju 2012), leading to high optical absorption and conductivity for photovoltaic applications (El-Nahass et al 2002). Recently, Santra and Kamat (2012) presented the first demonstrated strategy of transition metal- $\mathrm{Mn}^{2+}$ doping in single CdS and double-layered CdS/CdSe on a mesoporous $\mathrm{TiO}_{2}$ photoanode yielding improved efficiencies for both devices of $2.53 \%$ and $5.42 \%$, respectively, after $\mathrm{Mn}^{2+}$ doping. However, doping with metal elements was impaired by the thermal instability and induced more carrier recombinations in the cells. Consequently, doping with non-metal elements has been introduced to improve the photocatalytic efficiency, suppress the carrier recombination and modify its crystallization (Chen et al 2006). Previous literature, for instance, Luke et al (2008) have reported the synthesis of a CdSe semiconductor with nitrogen doping and has been deposited on a nanocrystalline $\mathrm{TiO}_{2}$ film for use as a photoelectrode, which had a power conversion efficiency of $0 \cdot 84 \%$ and it can be noted that combining nitrogen doping with the semiconductor sensitizer on $\mathrm{TiO}_{2}$ thin films is an effective and promising procedure to enhance the photoresponse in the near-UV and visible region. Liu et al (2013) successfully synthesized carbon quantum-dot-doped CdS microspheres (C/CdS), where the C/CdS exhibited a crucial role in enhancing the photocatalytic activity, efficient charge separation and transportation in the composites and furthermore, a higher and more stabilized photocurrent density was generated.

Previously, the study of Grey et al (1996) suggested that boron $\left(\mathrm{B}^{3+}\right)$-incorporated semiconductor-metal oxide material could improve the photocatalytic activity and act as photogenerated electron traps to facilitate the charge separation and also play an important role to suppress the recombination between photogenerated electrons and holes in the electrolyte region. However, there has been a little study of boron doping in semiconductor sensitizing, until now. Therefore, we were interested in the use of boron as a dopant in a semiconductor sensitizer to modify the characteristics and the electronic and photophysical properties of a sensitizer and to create a trap state in the midgap region of a sensitizer, leading to an alteration of the charge separation and recombination dynamics and to report on the synthesis of boron-doped manganese telluride (B-doped MnTe) semiconductor-decorated ZnO nanoparticles by the SILAR process. Their characteristics, optical and photovoltaic properties were investigated for SSC application as a novel tailoring sensitizer.

\section{Experimental}

\subsection{Preparation of $\mathrm{ZnO}$ photoelectrode}

$\mathrm{ZnO}$ powder ( $<100 \mathrm{~nm}$, Aldrich) was mixed with polyethylene glycol (PEG, $20 \mathrm{wt} \%$ ) and Triton X-100. The ratio of mixed materials to form a $\mathrm{ZnO}$ film was $\mathrm{ZnO}$ powder: PEG : Triton X-100 $=1: 2: 0 \cdot 1$ by weight and then, the solution was stirred for 30 min at room temperature. The $\mathrm{ZnO}$ photoelectrode was prepared by spreading on cleaned fluorine-doped tin oxide glass (FTO, $13 \Omega \mathrm{cm}^{-2}$, Aldrich) using a doctor blade technique. The as-prepared sample was dried at $120^{\circ} \mathrm{C}$ for $10 \mathrm{~min}$, then fired at $400{ }^{\circ} \mathrm{C}$ for $1 \mathrm{~h}$.

\subsection{Synthesis of B-doped MnTe sensitizer}

Non-metal B-doped MnTe sensitizer was synthesized on a $\mathrm{ZnO}$ photoelectrode through the two-step successive ionic layer adsorption and reaction (SILAR) process described below. First, $\mathrm{Mn}^{2+}$ ions were deposited on the $\mathrm{ZnO}$ surface by dipping the $\mathrm{ZnO}$ electrode into a precursor of $0.1 \mathrm{M} \mathrm{Mn}\left(\mathrm{NO}_{3}\right)_{2}$ with $0.075 \mathrm{M}$ boron powder in ethanol solution for $1 \mathrm{~min}$. Then, the electrode was rinsed thoroughly with ethanol and dried in air at $60{ }^{\circ} \mathrm{C}$ for $1 \mathrm{~min}$. Subsequently, the electrode was dipped into $0 \cdot 1 \mathrm{M}$ $\mathrm{Na}_{2} \mathrm{O}_{3} \mathrm{Te}$ methanol solution for another 1 min as a precursor for $\mathrm{Te}^{2-}$ ions, after which it was rinsed with methanol and dried again in air at $60{ }^{\circ} \mathrm{C}$ for another $1 \mathrm{~min}$. The entire process is referred to as one SILAR process. The $\operatorname{MnTe}(n)$ was referred to as $n$ SILAR cycles of the dipping process. When the number of SILAR cycles was increased by dipping the $\mathrm{ZnO}$ electrodes in the solution with boron doping, the $\mathrm{ZnO}$ electrodes became more black-brownish, compared with an uncoated sample and the samples are shown in figure 1.

\subsection{Characteristics of B-doped MnTe sensitizer}

The structural characteristics of the B-doped MnTe were investigated using a high-resolution field emission scanning electron microscope (FESEM, JEOL JSM 7001F) with an energy dispersive X-ray spectroscope (EDS, INCA PentaFET $\times 3$ Oxford). X-ray diffractometer (Model D8 


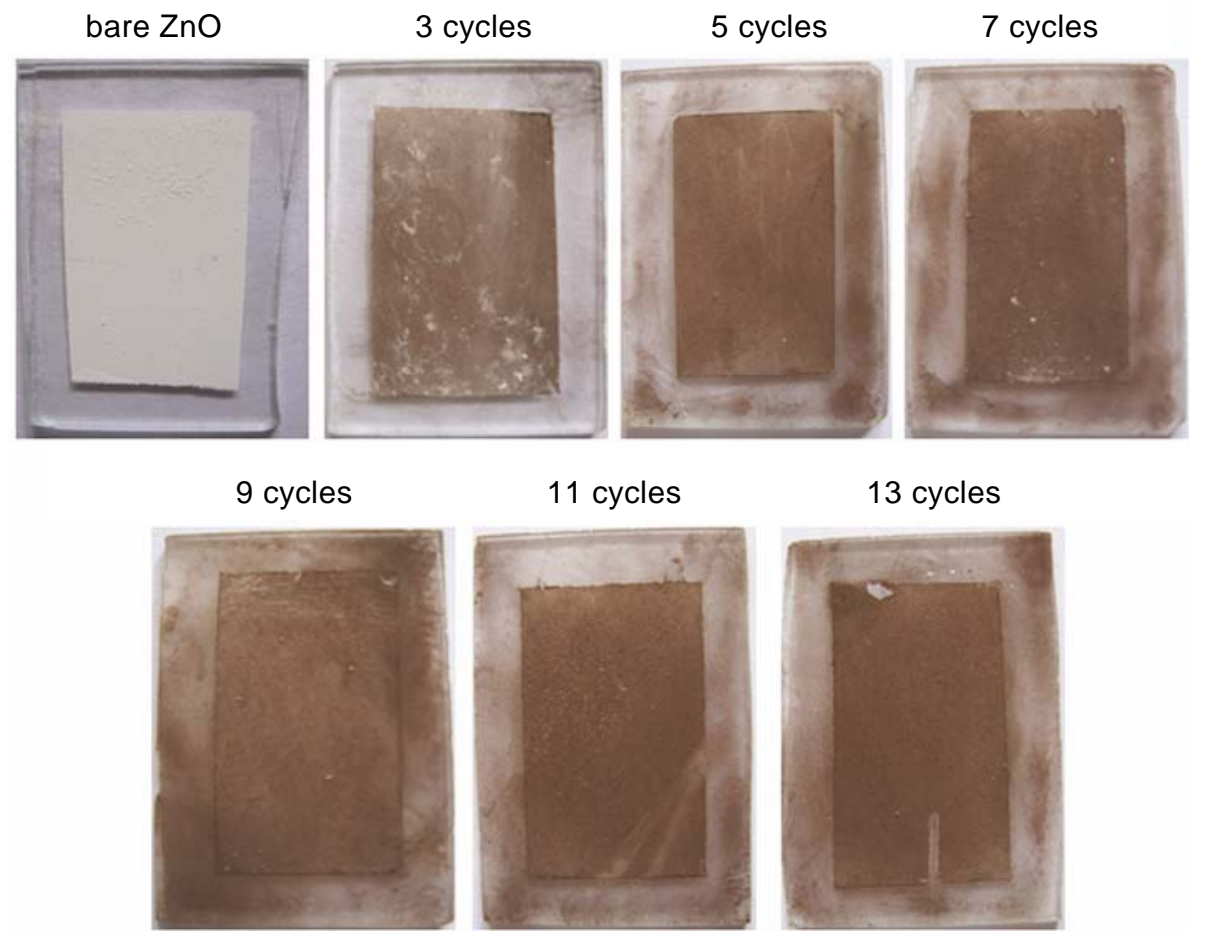

Figure 1. Characteristics of $\mathrm{ZnO}$ films after coating with various cycles of B-doped MnTe NPs.

Advance: Bruker AXS, Germany) was used to perform patterns of various conditions of photoelectrodes via CuK $\alpha$ radiation with a wavelength of $1.5406 \AA$, operated at $40 \mathrm{kV} / 30 \mathrm{~mA}$ from $20^{\circ}$ to $80^{\circ}$ and an increment of $0.01^{\circ}$. The scanning speed was $0 \cdot 3 \mathrm{~s}$ per step and a transmittance electron microscope (HRTEM, FEI-Techni-20 G' ${ }^{2}$ ) operating at $200 \mathrm{kV}$. The $\mathrm{ZnO}$ film with the B-doped MnTe sensitizer coating was prepared on a TEM copper grid using the procedure described in the previous section.

\subsection{Assembly of B-doped MnTe semiconductor-sensitized $\mathrm{ZnO}$ photoelectrode}

The B-doped MnTe semiconductor-sensitized ZnO photoelectrode was assembled with $\mathrm{Pt}$ counterelectrode (Pt $\mathrm{CE}$ ), prepared by $20 \mathrm{mM} \mathrm{Cl} \mathrm{H}_{2} \mathrm{Pt}$ dissolved in a mixed solution of acetone and $20 \%$ terpineol anhydrous and dropped on bare FTO glass, and then heated at $400{ }^{\circ} \mathrm{C}$ for $15 \mathrm{~min}$. A $190 \mu \mathrm{m}$ thick hot-melt parafilm was used as a spacer sealed between the photoelectrode and the counterelectrode. The iodide/triiodide $\left(\mathrm{I}^{-} / \mathrm{I}_{3}^{-}\right)$electrolyte consisted of $0.03 \mathrm{M} \mathrm{I}_{2}$ and $0.3 \mathrm{M} \mathrm{LiI}$ was dissolved in propylene carbonate and was injected through a pin hole in the $\mathrm{Pt}$ $\mathrm{CE}$ and each hole was then sealed.

\subsection{Optical and photovoltaic properties}

The absorption spectra of various conditions were recorded using a UV-Vis spectrometer (Lambda 25, Perkin Elmer).
The photovoltaic data were recorded under simulated sunlight from a solar simulator with radiant illumination of $100 \mathrm{~mW} / \mathrm{cm}^{2}$ (150 W Oriel Xe lamp with AM-1.5 filter) through a Keithley 2400 source meter. The incident light intensity was calibrated with a standard Si solar cell. The active area of the cell was $4 \times 4 \mathrm{~mm}$ and a metal mask of size $4.5 \times 4.5 \mathrm{~mm}$ was placed on top of the sample before recording the $I-V$ measurements.

\section{Results and discussion}

Figure 2(a) shows the SEM images of bare $\mathrm{ZnO}$ nanoparticles and the B-doped MnTe semiconductor coated on the $\mathrm{ZnO}$ nanoparticles is shown in figure 2(b). Furthermore, in figure 2(c), the TEM micrograph shows clearly the B-doped MnTe nanoparticle characteristics deposited on the $\mathrm{ZnO}$ surface with a diameter of $15-30 \mathrm{~nm}$. To confirm the deposition of B-doped MnTe semiconductors onto the $\mathrm{ZnO}$ surface, we investigated the EDS point analysis in figure 2(d) and the results showed the presence of manganese and tellurium on the $\mathrm{ZnO}$ surface; the stoichiometry based on the atomic ratio between the Mn and Te was approximately $1: 1$. The diffraction patterns in figure 3(a) show the $\mathrm{ZnO}$ signal before and after the B-doped MnTe coating. The signals of the two conditions looked similar and mainly show the $\mathrm{ZnO}$ signal, but the signal of $\mathrm{ZnO}$ after the B-doped MnTe coating was decreased. The resulting signal did not produce the peak of B-doped MnTe in the $\mathrm{ZnO}$ spectra due to the very 

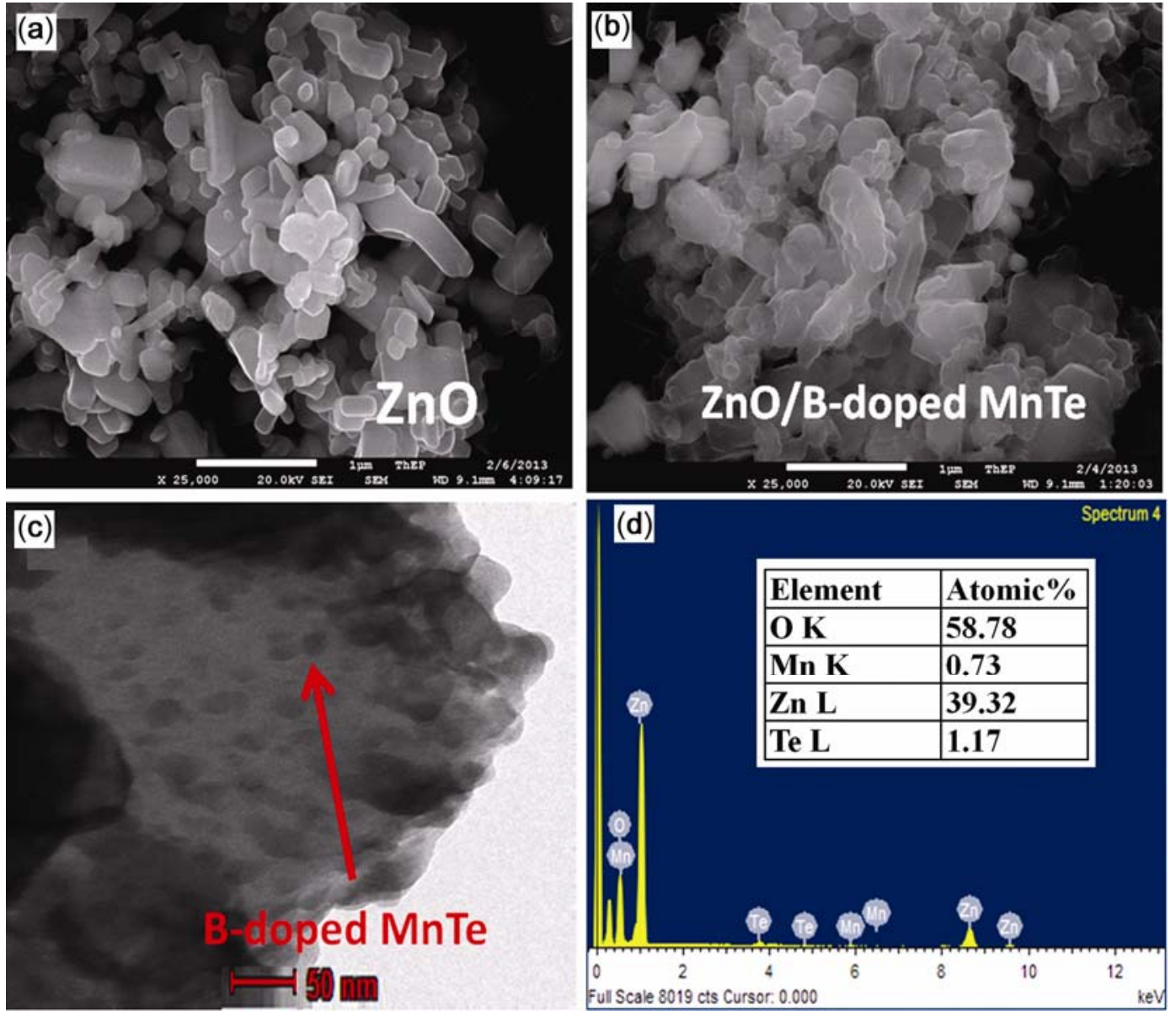

Figure 2. SEM images of (a) bare $\mathrm{ZnO}$, (b) B-doped MnTe-coated $\mathrm{ZnO}$, (c) TEM image of B-doped MnTe-coated ZnO and (d) EDS analysis and (inset) chemical elements of B-doped MnTe-coated ZnO nanoparticles.
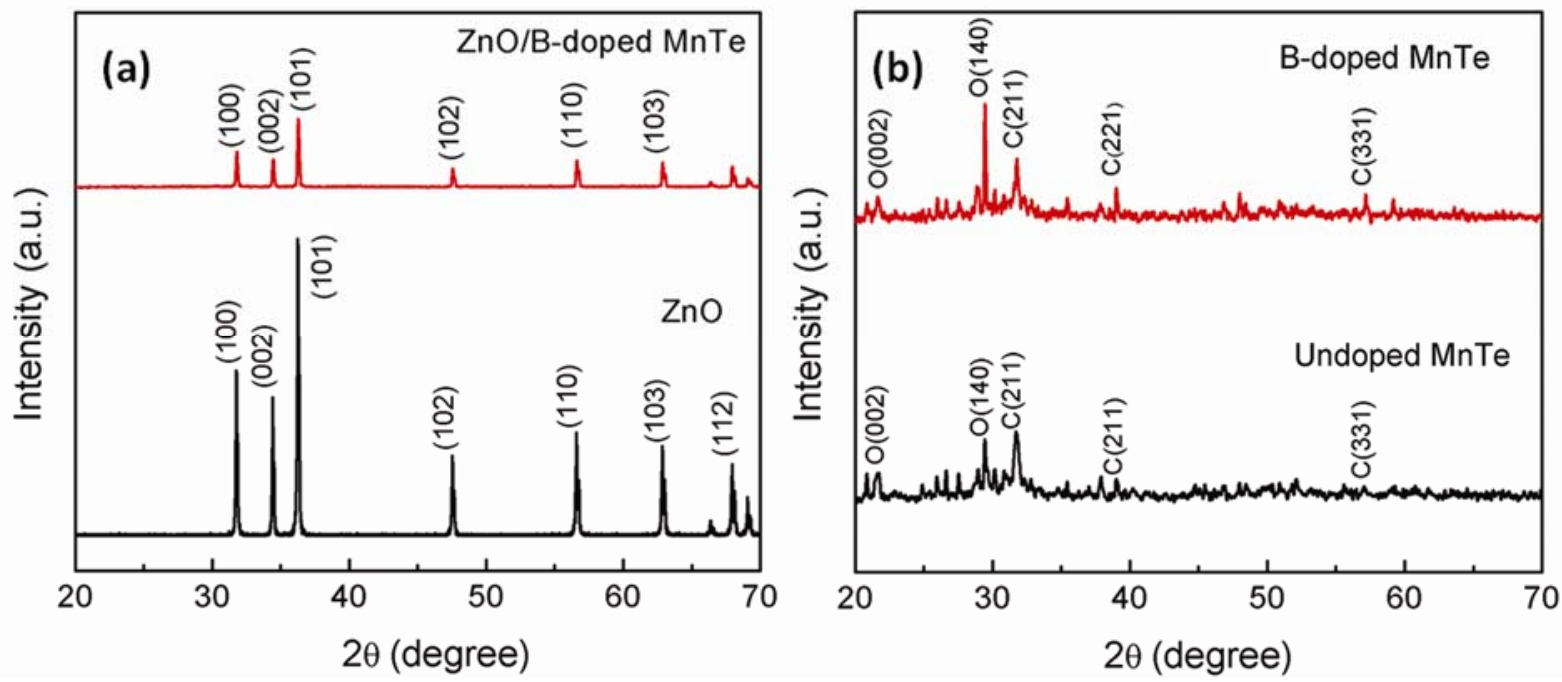

Figure 3. XRD patterns of (a) bare $\mathrm{ZnO}$ and B-doped MnTe-coated $\mathrm{ZnO}$, and (b) comparison between undoped and B-doped MnTe NPs.

low intensity compared to that of the $\mathrm{ZnO}$ signal. The as-synthesized MnTe nanoparticles before and after boron doping in figure 3(b) confirm that the structure is crystalline. The mixed growth phases both before and after B-doped MnTe coating, i.e. orthorhombic MnTe at $2 \theta=$ 21.707 ( 00 2 $)$ and 29.249 (1 4 0) (JCPDS no. 40-1195) 
and cubic $\mathrm{MnTe}_{2}$ at $2 \theta=31.538$ (2 111 ) and 38.882 (2 2 1) (JCPDS no. 73-1526), agreed with the results of Sharma et al (2007). However, while both spectra look

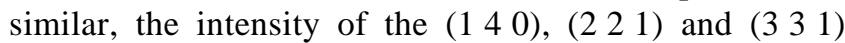
peaks were higher due to the improvement in the crystalline quality and the larger size of crystal after boron doping.

Figure 4(a) shows the optical properties of absorption of bare $\mathrm{ZnO}$, ZnO/undoped and B-doped MnTe NPs to support the crystal structures. The spectra showed that the samples had greater absorptivity to the incident light after the MnTe coating and in the B-doped MnTe structure. The plot of $(\alpha h v)^{2}$ vs $h v$ with various conditions was performed to determine the photon energy of the samples and showed the intercept of photon energy on the $X$-axis by extrapolating the linear line portion of the plot, producing values of $3.20 \mathrm{eV}$ for bare $\mathrm{ZnO}, 1.30 \mathrm{eV}$ for undoped MnTe NPs and 1.27 eV for B-doped MnTe NPs,


Figure 4. (a) Optical absorption characteristics and (b) $I-V$ curves for B-doped MnTe-coated $\mathrm{ZnO}$ with various SILAR cycles. which are in agreement with previous reports (Alvisatos 1996; Beard 2011). The narrower energy gap after boron doping was due to the larger size of the MnTe NPs deposited on the $\mathrm{ZnO}$ surface. Figure 4(b) shows the photovoltaic performance of various SILAR cycles of the B-doped MnTe NPs-deposited ZnO film. The shortcircuit current density $\left(J_{\mathrm{sc}}\right)$ was $0.03-0.08 \mathrm{~mA} \mathrm{~cm}^{-2}$, the open-circuit voltage $\left(V_{\text {oc }}\right)$ was $0 \cdot 17-0 \cdot 37 \mathrm{~V}$, the fill factor (FF) was $0 \cdot 25-0.74$ and the power conversion efficiency ( $\eta$ ) was $3-14 \times 10^{-3} \%$. The best $\eta$ value of $14 \times 10^{-3} \%$ was yielded at $n=11$ with $J_{\mathrm{sc}}=0.07 \mathrm{~mA} \mathrm{~cm}^{-2}, V_{\text {oc }}=0.26$ $\mathrm{V}$ and $F F=0 \cdot 74$. The undoped sample is shown for comparison in figure 5; the cell yielded a higher $J_{\text {sc }}$ value of $0.13 \mathrm{~mA} \mathrm{~cm}^{-2}$ and lower $V_{\text {oc }}$ and $F F$ values of $0.14 \mathrm{~V}$ and $0 \cdot 26$, respectively, with an $\eta$ value of $4 \times 10^{-3} \%$. Hence, the power conversion efficiency of the boron-doped MnTe coated on the $\mathrm{ZnO}$ photoelectrode was increased by approximately 3.5 times or $250 \%$, compared to that of the undoped sample. The percentage increase in the $J_{\text {sc }}$ value and the percentage reduction in the $V_{\text {ос }}$ value were $86 \%$ and $46 \%$, respectively, while the $F F$ value was reduced by $65 \%$ for the undoped sample, which arose from the large recombination of photogenerated electrons to the oxidized species at the photoelectrode and electrolyte interface (Goswami and Mandale 1978). There is little literature reporting the advantages of nonmetal-boron doping and interpreting the fact mechanism in figure 6, which is in such a good agreement with our results. The higher power conversion efficiency may be attributable to the trap state of boron doping as a midgap of MnTe NPs to facilitate the transfer of photogenerated electrons and low energetic electrons from the $E_{\mathrm{CB}}$ of MnTe to the $E_{\mathrm{CB}}$ of $\mathrm{ZnO}$, leading to an increase in the photocatalytic

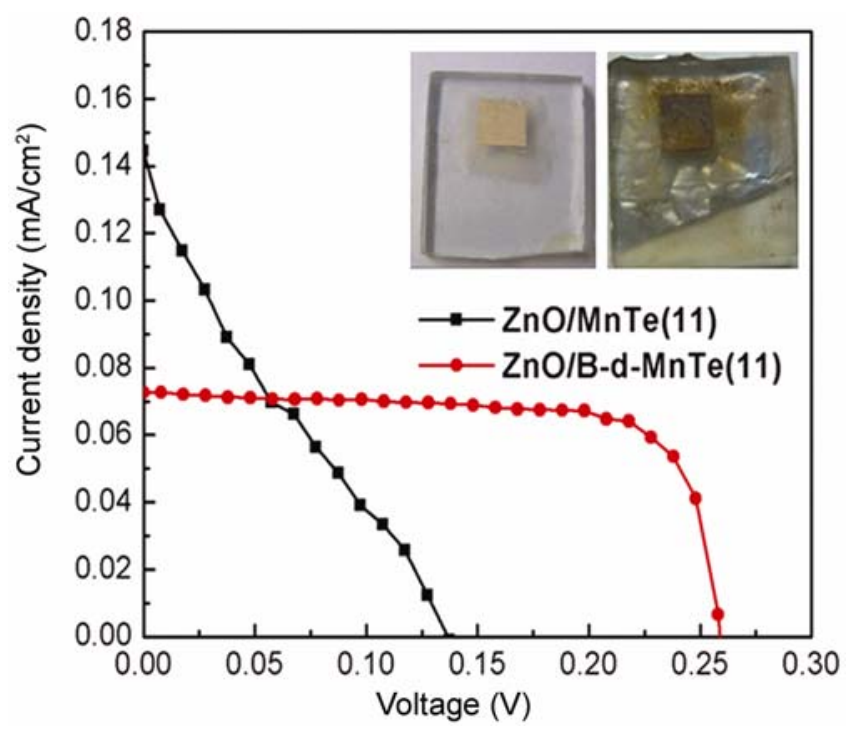

Figure 5. Comparison between undoped and B-doped MnTecoated $\mathrm{ZnO}$ with optimal SILAR cycle $(n=11)$ measured under $100 \mathrm{~mW} \mathrm{~cm}^{-2}$ (AM $1.5 \mathrm{G}$ ). 


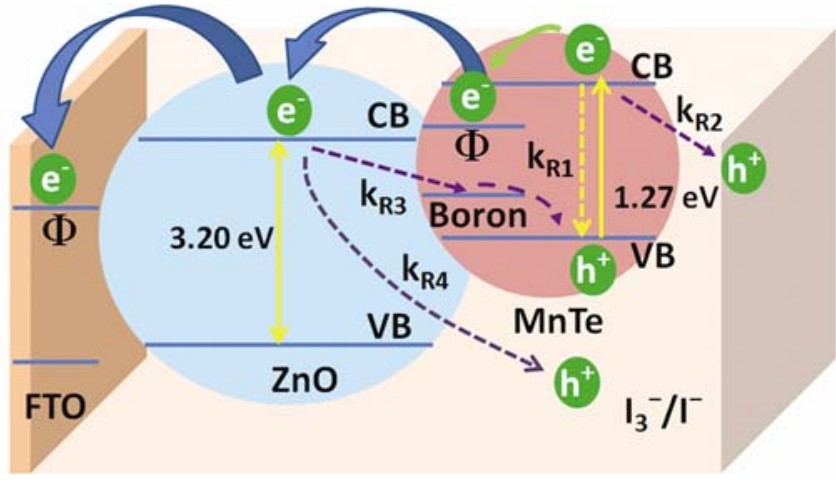

Figure 6. Schematic diagram of the carrier recombination and electron transfer process from B-doped MnTe into $\mathrm{ZnO}$ and through FTO.

activity and reduced carrier recombination inside the MnTe and between the MnTe/electrolyte interfaces (Grey et al 1996; Chen et al 2006). However, the low power conversion efficiency was considered to result from three components: (1) there are some regions of poor electrical contact between the B-doped MnTe NPs and the $\mathrm{ZnO}$ surface and between each $\mathrm{ZnO} \mathrm{NP}$ due to the $\mathrm{ZnO}$ NPs not being spherical in shape, which leads to the low rectifying properties of the cell and it is difficult for the electrons to move in the external circuit, caused from the roughened surfaces of the photoelectrode; (2) although the midgap of boron at the MnTe will facilitate the transport of photogenerated and low energetic electrons on the $E_{\mathrm{CB}}$ of the MnTe and move forward to the $E_{\mathrm{CB}}$ of $\mathrm{ZnO}$, there are still some carrier recombinations inside the MnTe structure $\left(k_{\mathrm{R} 1}\right)$ and at the MnTe and electrolyte interface $\left(k_{\mathrm{R} 2}\right)$; (3) there are no trap state mediator as a midgap to support electron transfer in the ZnO NPs; thus, it results in a high probability of carrier recombination at the $E_{\mathrm{VB}}$ of the $\mathrm{MnTe}\left(k_{\mathrm{R} 3}\right)$ and at the $\mathrm{ZnO}$ and electrolyte interface $\left(k_{\mathrm{R} 4}\right)$.

\section{Conclusions}

We synthesized novel B-doped binary semiconductor MnTe NPs-sensitized ZnO solar cells using the SILAR technique. The energy gap of the MnTe sensitizer became narrower after boron doping, which is advantageous for broadening the absorption from the visible region to the NIR region and for improving its photocatalytic activity due to the suppression of carrier recombinations via a trap state in the MnTe structure, leading to higher power conversion efficiency. The low power conversion efficiencies produced in this research need to be improved by using an optimal material as a passivation layer or by further treatment to suppress carrier recombination. The research on this issue represents a stage in the improvement to produce higher power conversion efficiency and greater stability based on this type of semiconductor nanoparticle as a sensitizer.

\section{Acknowledgements}

We would like to acknowledge the Department of Physics and Research Promotion and Technology Transfer Center (RPTTC), Faculty of Liberal Arts and Science at Kasetsart University, Kamphaeng Saen Campus, for financial support and thanks to the Kasetsart University Research and Development Institute (KURDI) for critical proof reading. Moreover, Auttasit Tubtimtae and Suwanna Sheangliw would like to thank Applied Physics Research Laboratory (APRL), Department of Physics and Material Sciences, Faculty of Science at Chiang Mai University, for providing materials and measurement facilities.

\section{References}

Alvisatos A P 1996 Science 271933

Beard M C 2011 J. Phys. Chem. Lett. 21282

Chao H Y, Cheng J H, Lu J Y, Chang Y H, Cheng C L and Chen Y F 2010 Superlattices Microstruct. 47160

Chen D, Yang D, Wang Q and Jiang Z 2006 Ind. Eng. Chem. Res. 454110

Chen J, Wu J, Lei W, Song J L, Deng W Q and Sun X W 2010 Appl. Surf. Sci. 2567438

Cheng K C, Law W C, Yong K T, Nevins J S, Watson D F, Ho H P and Prasad P N 2011 Chem. Phys. Lett. 515254

Deepa K G and Nagaraju J 2012 Mater. Sci. Eng. B177 1023

El-Nahass M M, Zeyada H M, Aziz M S and El-Ghamaz N A 2002 Opt. Mater. 20159

Ganesh T, Mane R S, Cai G, Chang J H and Han S H 2009 J. Phys. Chem. C113 7666

Georgekutty R, Seery M K and Pillai S C 2008 J. Phys. Chem. C112 13563

Goswami A and Mandale A B 1978 Jpn. J. Appl. Phys. 17473

Grey I E, Li C and Macrae C 1996 J. Solid State Chem. 127240

Hamanaka Y, Ogawa T and Tsuzuki M 2011 J. Phys. Chem. C 1151786

Hu L and Chen G 2007 Nano Lett. 73249

Kahn M L, Monge M, Collière V, Senocq F, Maisonnat A and Chaudret B 2005 Adv. Funct. Mater. 15458

Kamat P V 2008 J. Phys. Chem. C112 18737

Kongkanand A, Tvrdy K, Takechi K, Kuno M and Kamat P 2008 J. Am. Chem. Soc. 1304007

Lin M C and Lee M W 2011 Electrochem. Commun. 131376

Liu Y, Yu Y X and Zhang W D 2013 J. Alloys Compd. 569102

Luke T L, Wolcott A, Xu L P, Chen S, Wen Z, Li J, Rosa E D L and Zhang J Z 2008 J. Phys. Chem. C112 1282

Majidi H and Baxter J B 2011 Electrochim. Acta 562703

Miyauchi M 2011 Chem. Phys. Lett. 514151

Mu L X, Shi W S, Zhang T P, Zhang H Y, Wang Y, She G W, Gao Y H, Wang P F, Chang J C and Lee S T 2011 Appl. Phys. Lett. 98163101

Murray C B, Kagan C R and Bawendi M G 2000 Annu. Rev. Mater. Sci. 30545 
Okazaki K, Kubo K, Shimogaki T, Nakamura D, Higashihata M and Okada T 2011 Adv. Mater. Lett. 2354

Oleszkiewicz J, Kisiel A and Ignatowicz S A 1988 Thin Solid Films 1571

Panda S K, Gorai S and Chaudhuri S 2006 Mater. Sci. Eng. B129 265

Potter Jr B G and Simmons J H 1988 Phys. Rev. B37 838

Ravirajan P, Peiró A M, Nazeeruddin M K, Gräetzel M, Bradley D D C, Durrant J R and Nelson J 2006 J. Phys. Chem. B110 7635

Samal A K and Pradeep T 2010 J. Phys. Chem. C114 1796

Santra P K and Kamat P V 2012 J. Am. Chem. Soc. 134 2508
Sharma R K, Singh G, Shul Y G and Kim H 2007 Physica B390 314

Tubtimtae A, Lee M W and Wang G J 2011 J. Power Sources 1966603

Tubtimtae A, Wu K L, Tung H Y, Lee M W and Wang G J 2010 Electrochem. Commun. 121158

Vogel R, Hoyer P and Weller H 1994 J. Phys. Chem. 983183

Vogel R, Pohl K and Weller H 1990 Chem. Phys. Lett. 174241

Wang G, Yang X, Qian F, Zhang J Z and Li Y 2010 Nano Lett. 101088

Yu W, Qu L, Guo W and Peng X 2003 Chem. Mater. 152854

Zhu G, Lv T, Pan L, Sun Z and Sun C 2011 J. Alloys Compd. $\mathbf{5 0 9} 362$ 